\title{
Influence of Iron Catalyst in the Carbon Spheres Synthesis for Energy and Electrochemical Applications
}

\author{
Manuela Scarselli, * Francesca Limosani, Maurizio Passacantando, Franco D'Orazio, \\ Michele Nardone, Ilaria Cacciotti, Fabiana Arduini, Eric Gautron, and Maurizio De Crescenzi
}

Carbon spheres of nanometric dimension are known since the first studies on the synthesis of fullerenes. Their shape originates from the curvature of a carbon sheet similar to fullerenes, but with numerous graphitic rings that regulate the inside structure and the formation of open edges at the surface. This paper focuses on the structural and electronic characterization of carbon spheres obtained from a chemical vapor deposition synthesis process. Two different sets of samples are analyzed in detail, in particular, electron microscopies and Raman spectroscopy help understanding the morphology and the graphitic-sp ${ }^{2}$ arrangement of the carbon atoms in the architectures. In addition, the iron catalyst used during the reaction process confers the carbon spheres a ferromagnetic response at room temperature. Therefore, both the structural properties of the samples and the active contribution of iron mark the difference in the measured photoresponse as well as in the electrochemical behavior. The X-ray photoelectron spectroscopy study addresses these points by giving information on the composition and the iron chemical state in the assembly. The collected results underline the advantages offered by this nanomaterial for sustainable applications.

\section{Introduction}

The discovery of fullerenes ${ }^{[1]}$ marked the beginning of a new era for carbon allotropes of nanometer dimension, as they are the first cage-like structure with a surface curvature that originates from the insertion of pentagonal carbon rings into the hexagonal graphene layer. Since then, the scientific community started the investigation on other curved carbon nanostructures that could enrich the current knowledge of the structural and electronic properties of quantum sizerelated materials. Added to this, there is a strong interest in producing small-scale materials for technological applications. ${ }^{[2]}$ Before fullerenes, the most studied and well-characterized spherical carbon materials were carbon blacks, ${ }^{[3,4]}$ and their properties found several industrial applications. ${ }^{[5]}$ Different from fullerenes, the carbon spheres (CSs) have an internal structure constituted of numerous gra-

Dr. M. Scarselli, Prof. M. De Crescenzi

Dipartimento di Fisica

Università di Roma Tor Vergata

Via della Ricerca Scientifica 1, 00133 Roma, Italy

E-mail: manuela.scarselli@roma2.infn.it

Dr. F. Limosani, Dr. F. Arduini

Dipartimento di Scienze e Tecnologie Chimiche

Università di Roma Tor Vergata

Via della Ricerca Scientifica 1, 00133 Roma, Italy

Prof. M. Passacantando, Prof. F. D'Orazio, Prof. M. Nardone

Dipartimento di Scienze Fisiche e Chimiche

Università degli Studi dell'Aquila

Via Vetoio, 67100 Coppito, L'Aquila, Italy

Prof. I. Cacciotti

Dipartimento di Ingegneria

Università di Roma Niccolò Cusano (INSTM-UdR)

Via Don Carlo Gnocchi 3, 00166 Roma, Italy

Dr. E. Gautron

Institut des Matériaux Jean Rouxel

IMN

Universitè de Nantes

CNRS

44322 Nantes, France

The ORCID identification number(s) for the author(s) of this article can be found under https://doi.org/10.1002/admi.201800070.

DOI: 10.1002/admi.201800070 phitic sheets that form mostly closed shells but also waving flakes whose open edges yield reaction sites located in the outer surface. Spherically shaped carbon materials, not including the fullerene family, have been given many names; these include carbon balls, carbon nanospheres, carbon microbeads, carbon blacks, onions, and others. It is clear that properties like size, morphology, and inner structure can vary substantially, nevertheless the generic term CSs applies to all carbon materials that have a spherical or near spherical shape. ${ }^{[6-8]}$ The CS structures can be classified according to the size, degree of graphitization, and the resulting orientation of the carbon layers in the texture: concentric, radial, or random. ${ }^{8]}$ Therefore, they can be distinguished in: 1) carbon onions, with well-organized inner structure and diameter ranging between 2 and $20 \mathrm{~nm}$; 2) carbon spheres, less graphitized structures with diameters between 50 and $1000 \mathrm{~nm}$, and 3) carbon beads, that can have diameters up to tents of micrometers with a poor graphitization degree. Generally, each CS morphology derives from the synthesis route used. $^{[6,7]}$ In the last decades, some synthesis processes adopted for the production of carbon nanotubes, led by chance many side products including $\mathrm{CSS}_{.}{ }^{[9]}$ Therefore, some of these routes have been adapted to obtain CS as the main product but it is difficult to identify the best method since some of the parameters that regulate the growth of other carbon nanostructures ${ }^{[6,7]}$ 

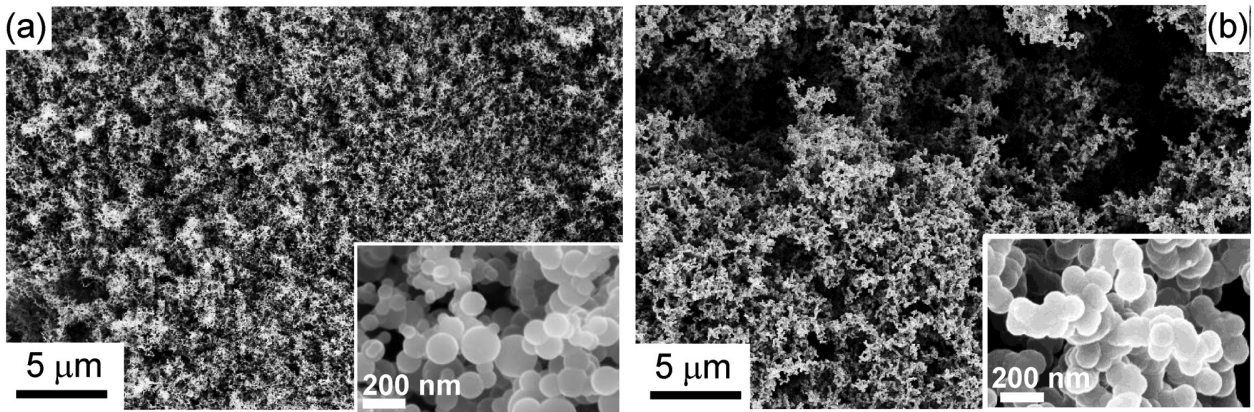

Figure 1. SEM micrographs of CS samples. a) Sample A, on large scale no side products or other carbon species are present, scale bar is $5 \mu$ m. The image in the inset shows well-defined CSs of different diameter, scale bar is $200 \mathrm{~nm}$. b) Sample B has similar behavior of the sample A on a large scale image, scale bar is $5 \mu \mathrm{m}$, while in the better resolved image in the inset the CSs have homogenous dimensions but some of them are partially welded, scale bar is $200 \mathrm{~nm}$.

seem to be less specific for the CSs. The key target is to ensure that the CSs are monodispersed and the outer surface properties are controlled. Several published reviews give an exhaustive description of the most significant CS synthesis routes ${ }^{[6,7]}$ including arc-discharge, ${ }^{[10]}$ laser ablation, ${ }^{[11]}$ chemical vapor deposition (CVD), ${ }^{[12]}$ and thermal pyrolysis. ${ }^{[13]}$ In detail, the arc-discharge and laser ablation methods require high energies and temperature for the synthesis and produce CSs of variable dimension. In addition, the yields tend to be low and the final materials require additional purification routes before their use. In the framework of alternative and new synthesis processes the CVD turns out to be the most flexible and relatively low cost technique for an efficient and controlled production of carbon species. ${ }^{[6,7,12]}$ In the process, a volatile carbon source decomposes and the carbon atoms rearrange to give well-organized carbon crystalline nanostructures. This method is versatile because it is able to produce CSs with well-defined characteristics changing some of the growth parameters such as the carbon source, the temperature, and the gas pressure. It is also possible to add a metal catalyst in the reaction for lessening the growth conditions, favor the dissociation of the carbon precursor and the formation of the graphene sheets that will constitute the CSs. ${ }^{[14]}$ The CSs are synthesized using a catalytic carbonization of methane over rare earth metal oxides or iron at a temperature higher than $1000{ }^{\circ} \mathrm{C},{ }^{[15]}$ or using high pressure processes $(30 \mathrm{MPa})$, like the carbonization of polyethylenepoly(vinyl chloride), ${ }^{[16]}$ and in carbon vapor from the decomposition of $\mathrm{b}-\mathrm{SiC}$ powder. ${ }^{[17]}$ Ferrocene as catalyst and different carbon precursors have been used in CVD operating at temperatures ranging from 900 to $1000{ }^{\circ} \mathrm{C} .{ }^{[18]} \mathrm{CSs}$ of variable size were obtained at relatively low temperature $\left(650^{\circ} \mathrm{C}\right)$ from the decomposition of acetylene over transition metal salts supported on kaolin substrate, the CSs obtained have variable size range with some side products like carbon nanotubes. ${ }^{[19]}$ Other processes, like hydrothermal, reduction, and template routes, gave good results, as described in detail in the literature. ${ }^{[6,7]}$ This paper first illustrates the a CVD often referred as floating catalyst process, in which both the iron catalyst and a carbon precursor are dispersed in a solution. Subsequently, drops of solution are injected into the hot zone of a furnace with the help of a gas carrier (argon) and an additional carbon gas precursor (acetylene). When the main elements (catalyst and carbon precursors) reach the high temperature zone, decompose in the elemental form, and the production of carbon species starts. The main advantages offered by this process are: 1 ) the synthesis dos not require any substrate, 2) the final yield is proportional to the reaction time, 3) there are few side products coming from the reaction, 4) the CSs have a narrow size distribution, 5) the reaction temperature is relatively low $750-800{ }^{\circ} \mathrm{C}$ ) compared to other synthesis routes, ${ }^{[6,7]}$ and 6) the production has a relatively low price. Mostly importantly, the encapsulation of catalyst particles in the spheres can be exploited in some applications, as illustrated in this paper. In particular, this paper focuses on the synthesis of two different sets of CSs obtained from the floating catalyst CVD process. These were subjected to different characterization techniques including scanning electron microscopy (SEM), X-ray diffraction (XRD), Raman spectroscopy, and X-ray photoelectron spectroscopy in order to qualify their structure, morphology, and chemical composition. The use of iron as catalyst during the synthesis confers to the CSs magnetic properties as demonstrated by magnetic characterization. Finally, magnetic and nonmagnetic CSs are challenged for energy and electrochemical applications demonstrating the improved performances of magnetic CSs and thus the advantage of using Fe as catalyst.

\section{Results and Discussion}

The experimental results illustrated in this paragraph were collected from two different carbon sphere samples. The first, namely sample A, was obtained from a synthesis process maintaining the argon/acetylene gas flux of $50 \mathrm{sccm} / 25 \mathrm{sccm}$, while for the second sample, named $\mathrm{B}$, the argon/acetylene gas flux was kept at $250 \mathrm{sccm} / 40 \mathrm{sccm}$ (see "Preparation of Carbon Spheres" section in the Experimental Section). The reaction time, the solution concentration, the injection rate in the hot zone, and the growth time were the same for the two processes.

\subsection{Characterization of Carbon Spheres}

The morphology of the CS samples was first investigated through systematic SEM measurements (Figure 1). In particular, Figure 1a reports a low and high magnification (inset) images of sample A. The low magnification evidences the 

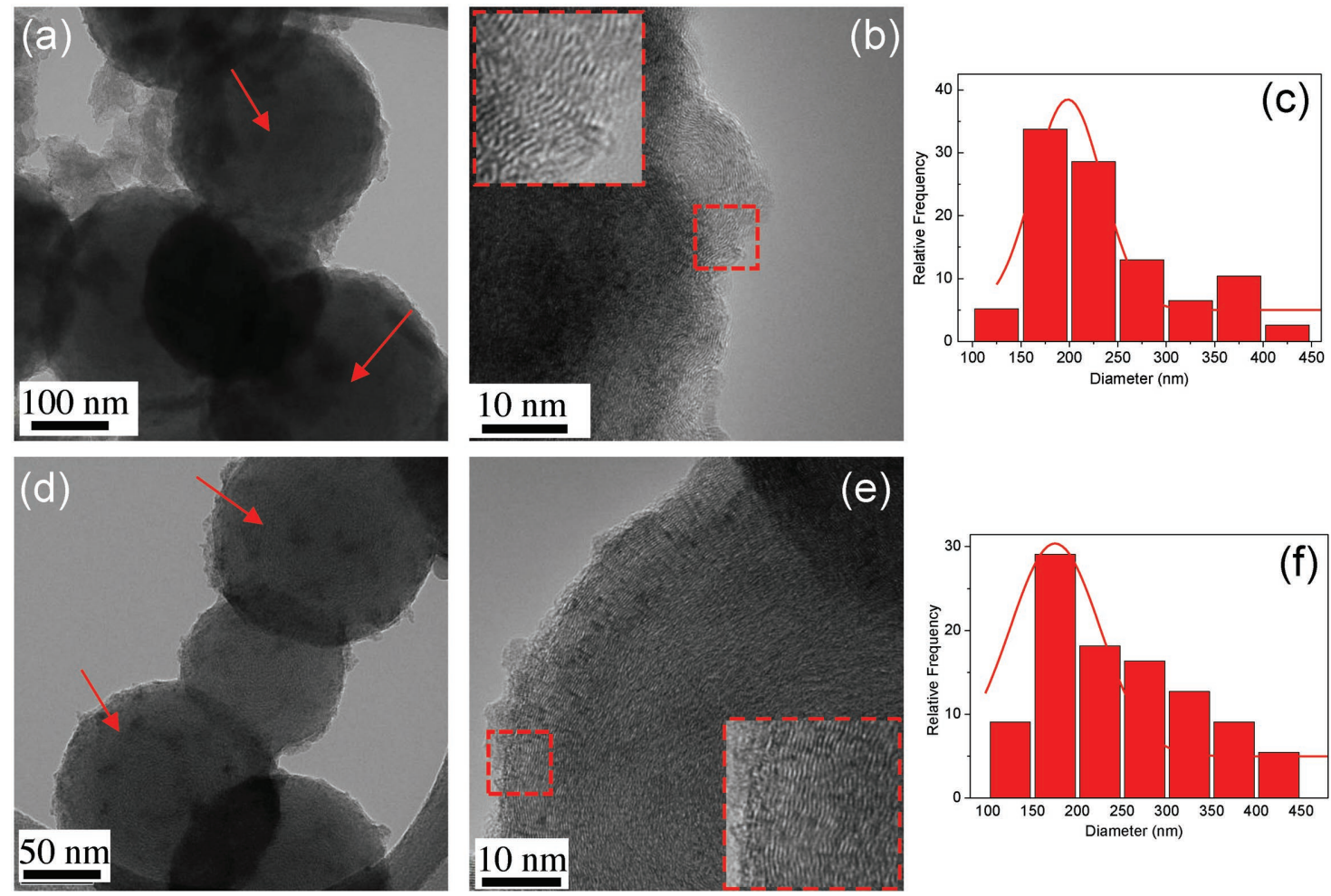

Figure 2. TEM images of samples A and B. Sample A: a) Large scale image shows CSs and residual side products. b) Disordered graphitic planes are present inside and outside the sphere forming open edges (inset). c) Size distribution histogram. Sample B: d) Large scale image shows the presence of CSs that form a necklace structure. e) The graphitic planes are ordered inside the sphere and the outer surface is not smooth (inset). $f$ ) Size distribution diagram. Red arrows in (a) and (d) point to clusters inside the spheres of different electronic contrast.

sample homogeinity and the absence of carbonaceous side products, while the inset shows well-defined carbon spheres of variable diameter. Similarly, Figure $1 \mathrm{~b}$ reports a combination of low and magnification images collected from sample B. No side products or other carbon species appear in the extended image, and the inset exhibits that these spheres have homogeneous average size but sometimes are partially welded.

The transmission electron microscopy (TEM) helped investigating the inner and the outer surface structure of both samples. Figure 2 shows a collection of images of few carbon spheres dispersed on the TEM grid from samples A and B. In particular, Figure $2 \mathrm{a}-\mathrm{c}$ provides some insight into the structure of the spheres from sample A. The inner part is constituted of disordered graphitic sheets while the outer surface is not regular in shape evidencing many protruding graphitic planes that make the surface very defective (Figure $2 b$ ). The statistical analysis of the diameter distribution reported in Figure 2c gives an average CS diameter of $200 \pm 10 \mathrm{~nm}$.

Similarly, Figure $2 \mathrm{~d}-\mathrm{f}$ reports the TEM images obtained from sample B. The CSs are composed of graphene layers that, differently from sample A, are more uniformly distributed inside the spheres (Figure 2e) at a relative average distance that ranges between 0.33 and $0.35 \mathrm{~nm}$, compatible with that of crystal layers in graphite. The outer surface is more regular and smooth compared to sample A. The average diameter found for this sample from the statistical analysis is $175 \pm 10 \mathrm{~nm}$ (Figure 2f).

From SEM and TEM studies, we conclude that each synthesis process influenced the inner organization of the graphite sheets that compose the CSs and, mostly important, the outer surface structure, while the average diameter is comparable. The similarity in the sphere size found derives from the identical reaction time of process. The carbon $\mathrm{sp}^{2}$ hybridization in the sphere assembly comes both the electron energy loss spectra (EELS) and Raman spectra (Figures S1 and S2, Supporting Information). The EELS spectra show two typical $\mathrm{sp}^{2}$ features similar to that of graphite plasmon losses but located at lower energy position because of the reduced dimension of the material (Figure S1, Supporting Information). The two main Raman features the D (around $1350 \mathrm{~cm}^{-1}$ ) and $\mathrm{G}$ bands (around $1580 \mathrm{~cm}^{-1}$ ) that originate from the carbon defects and $\mathrm{sp}^{2}$ vibrational modes, respectively, highlight a high number of structural imperfections and evidence a not perfect degree of graphitization for both samples A and B (Figure S2, Supporting Information). These results are comparable to those reported for similar CSs. ${ }^{[10,17-19]}$

Interestingly, from the TEM images smaller points of different electronic contrast appear inside the CSs. The arrows in Figures 2a,d highlight some of them. These probably consist of iron catalyst residual particles encapsulated during the reaction process. In order to deepen this point, we followed different routes. First, the presence of catalyst residues in the reaction products can be easily appreciated at the macroscopic level. In fact, when a permanent magnet is approached to a vial containing the CS powder some of it is lifted under the action of the magnet, clearly demonstrating that a portion of the reaction products has a significant magnetic moment, whereas the 


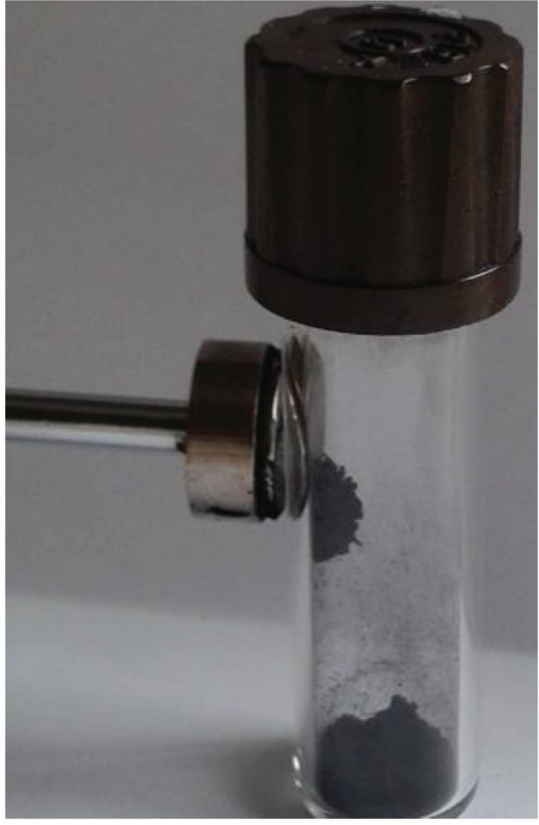

Figure 3. Image of the magnetic separation of one of the synthesis products. Each sample powder under the action of the permanent magnet shares into two parts, magnetic and nonmagnetic one, respectively.

rest of the material is much more weakly magnetic (Figure 3). The observed behavior can only originate from the iron participating to the reaction, since it is the only magnetic element exploited in the synthesis.

Therefore, each sample powder was separated into two parts: one magnetic that corresponds to the material that responds to the external action of the permanent magnet and the second part that is the residual product left at the bottom of the vial. The separation was performed for both samples, that from now on will be named $A_{m}, B_{m}$ (magnetic part) and $A_{n m}, B_{n m}$ (nonmagnetic), respectively.
Table 1. Concentration from fit analysis of the different Fe coordination.

\begin{tabular}{lccc}
\hline Sample & \multicolumn{3}{c}{ Concentration from fit analysis [\%] } \\
\hline & $\mathrm{Fe}^{0}$ & $\mathrm{Fe}^{2+}$ & $\mathrm{Fe}^{3+}$ \\
$\mathrm{B}_{\mathrm{m}}$ & 54 & 20 & 26 \\
$\mathrm{~B}_{\mathrm{nm}}$ & - & - & 100 \\
\hline
\end{tabular}

X-ray photoelectron spectroscopy (XPS) analysis was performed on the two samples A (red lines) and B (black lines), distinguishing between the magnetic and nonmagnetic part. The extended spectral region (see Figure S3, Supporting Information) scans display the contribution coming from the main elements involved in the reaction process for both the samples: carbon, iron, oxygen, and chloride. The Fe $2 p$ peak was investigated in detail and the spectra are reported in Figure 4.

The iron intensity signal from the magnetic and nonmagnetic parts of sample A (red lines) is very low and nearly identical (Figure 4a). In addition, the binding energy comes from the stoichiometric $\mathrm{Fe}_{2} \mathrm{O}_{3}$ oxide rather than metallic Fe 2p. The spectra obtained from the magnetic part of sample $B\left(B_{m}\right.$, black line) has a marked contribution coming from metallic Fe. Figure $4 \mathrm{~b}, \mathrm{c}$ shows the results of the deconvolution of the Fe2p electron core-level of the samples $\mathrm{B}_{\mathrm{m}}$ and $\mathrm{B}_{\mathrm{nm}}$, respectively. The peak positions of $\mathrm{Fe} 2 \mathrm{p}_{3 / 2}$ and $2 \mathrm{p}_{1 / 2}$ depend on the ionic state of Fe. In particular, we have considered, for the fit procedure, three different ionic states identified by: $\mathrm{Fe}^{0}$ (metallic state), $\mathrm{Fe}^{2+}$, and $\mathrm{Fe}^{3+}$ (oxide state) associated with the satellite peaks that are sensitive to the oxidation states. Table 1 reports the concentration of the Fe coordination states for the $B_{m}$ and $B_{n m}$ samples. Therefore, from the obtained results, if we assume that iron is mostly contained inside the CS samples, it oxidized during the reaction process.

The XRD patterns gave additional information on the samples as reported in Figure S4 in the Supporting Information, in which the graphite structure presents a strong dependence with the sample type. In particular, the sample $B_{m}$ shows a better

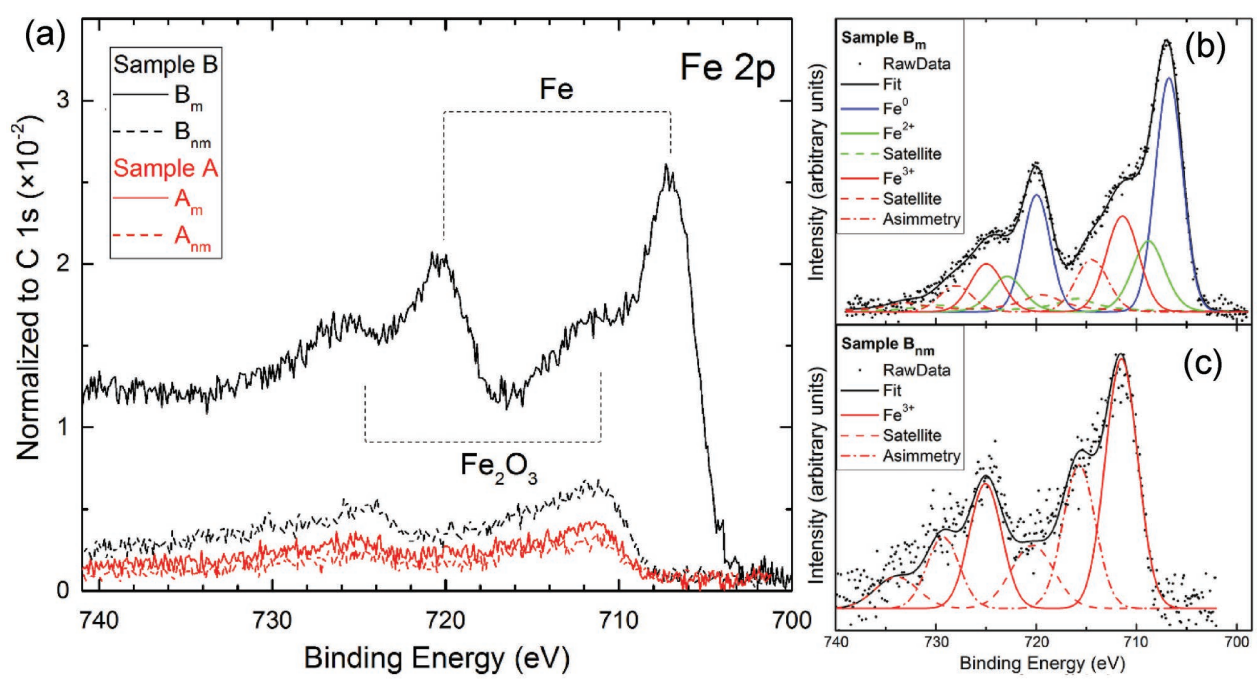

Figure 4. XPS regional scan of iron showing the deconvolution of the Fe2p peak. a) Sample $A_{m}$ (red line) and $A_{n m}$ (red dashed line) and sample $B_{m}$ (black line) and $B_{n m}$ (black dashed line). Spectral lines were normalized to the Cls signal. Panels (b) and (c) show the fitting analysis of the samples $B_{m}$ and $B_{n m}$, respectively, to check the different coordination state of Fe. 

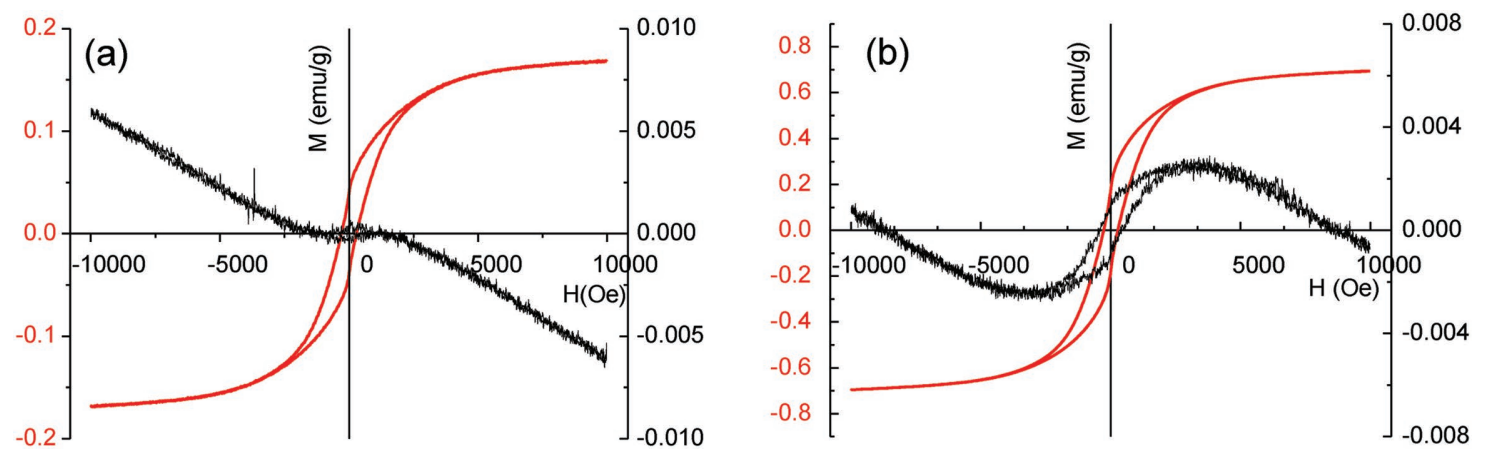

Figure 5. Magnetization curves of a) sample A and b) sample B. Red (black) curves refer to the magnetic (nonmagnetic) part of the samples. Notice that the corresponding ordinate axes (magnetic moment per unit mass) have different scales for the magnetic (left scale) and nonmagnetic (right scale) components of the specimen. The negative asymptotic slope of the nonmagnetic plots is due to the diamagnetic contribution of the carbon spheres, which becomes relevant when the ferromagnetic signal is very small.

graphitization (graphite with the conventional AB stacking) and the presence of an initial structuring of the diffraction pattern from the catalytic nanoparticles of small size associated to $\alpha$-Fe, $\mathrm{Fe}_{3} \mathrm{C}$, and graphite-2H phases.

The hysteresis loops, acquired at room temperature, allowed probing the magnetic properties of the samples. Figure $\mathbf{5}$ reports the magnetization curves of the two portions of samples A (Figure 5a) and B (Figure 5b). Red (black) curves refer to the magnetic (nonmagnetic) part of the samples. Notice that the corresponding ordinate axes (magnetic moment per unit mass) have different scales for the magnetic (left scale) and nonmagnetic (right scale) components of the specimen. In all the plots of the (diamagnetic) signal from the probe and from the sample holder is already subtracted.

Therefore, the negative asymptotic slope observed for the nonmagnetic plots is due to the diamagnetic contribution of the carbon spheres, which becomes relevant when the ferromagnetic signal is very small. On the other hand, the incomplete saturation even at magnetic fields of 1T, detected for the magnetic samples, can be ascribed to the iron-based particle size distribution. In fact, larger size particles (or even more elongated ones) have sufficient magnetic anisotropy to possess a permanent and nonfluctuating magnetic moment, giving rise to hysteresis, whereas clusters with smaller size are in a superparamagnetic regime and contribute to the magnetization loop with a Langevin-like function. ${ }^{[20]}$

Table 2 reports the main magnetic parameters extrapolated from the four hysteresis loops: the coercive field (column 2), the estimated saturation magnetic moment per unit mass (column 3), and the number of magnetic iron atoms per unit sample mass (column 4) calculated from column 3, assuming that the magnetic moment for a Fe atom is 1.2 bohr magnetons, a value compatible for compounds such as maghemite

Table 2. Magnetic measurements results for the four CS samples.

\begin{tabular}{lccc}
\hline Sample & $H_{c}[\mathrm{Oe}]$ & $M_{\mathrm{s}}\left[\mathrm{emu} \mathrm{g}^{-1}\right]$ & $N_{\mathrm{Fe}}[$ atoms g \\
\hline$A_{m}$ & 290 & 0.17 & $1.4 \times 10^{19}$ \\
$A_{n m}$ & 330 & 0.0018 & $1.5 \times 10^{17}$ \\
$B_{m}$ & 270 & 0.70 & $5.8 \times 10^{19}$ \\
$B_{n m}$ & 300 & 0.0053 & $4.4 \times 10^{17}$ \\
\hline
\end{tabular}

$\left(\gamma\right.$ - $\left.\mathrm{Fe}_{2} \mathrm{O}_{3}\right)$, magnetite $\left(\mathrm{Fe}_{3} \mathrm{O}_{4}\right)$, and cementite $\left(\mathrm{Fe}_{3} \mathrm{C}\right)$. The last compound is clearly revealed in the X-ray diffraction analysis of sample $\mathrm{B}_{\mathrm{m}}$ (see Figure S4, Supporting Information) and already associated to a superparamagnetic response when in nanoparticle form. ${ }^{[21,22]}$ An accurate comparison with the XPS data, the amount of magnetic iron with respect to the overall $\mathrm{Fe}$ content, is about one order of magnitude less, for the magnetic parts of the two samples. Therefore, only a small fraction of the iron content exhibits an appreciable magnetic response. This is not surprising if we consider that even the residual powder not selected by the magnetic separation method (namely, samples $B_{n m}$ and $A_{n m}$ ), contains comparable amount of iron with respect to their magnetic counterparts.

Observing more accurately the magnetic results, we note that the shapes of the hysteresis loops of samples A and B are very similar, although not identical. However, the magnetic response of sample B is much higher than that of sample A by a factor around four for the magnetic portions. This is in agreement with a greater iron content in sample B detected by XPS (by a factor of about six). The measured coercive field $\left(H_{c}\right)$ for the samples is comparable to that reported in the literature for other Fe filled carbon species like multiwalled nanotubes. ${ }^{[23,24]}$

From the results illustrated, a possible mechanism for the formation of CSs can be inferred. In the reaction process, the iron has the role of catalyzing the synthesis of carbon spheres and activates the carbon atoms produced by the dissociation of acetylene $\mathrm{C}_{2} \mathrm{H}_{2}$ and the other carbon-containing species. In the hot zone, the iron atoms aggregate into nanoparticles that are later encapsulated during the formation of quasi-spherical carbon shells. With increasing reaction time, the Fe nanoparticles present in the carbon shells become smaller and can delocalize in the carbon sheets eventually leaving the spheres that have formed. Therefore, it is possible that Fe is not always present in the CSs or its concentration is below the detection limit. In addition, there is a dependence of the CSs final structure upon the gas flow rates, being all the other parameters left unchanged (reaction temperature, and time, ferrocene concentration, solvent, cooling down time). In particular, the $\mathrm{Ar} / \mathrm{C}_{2} \mathrm{H}_{2}$ changes the average relative speed of the carbon atoms and $\mathrm{Fe}$ nanoparticles in the reaction zone. The acetylene gas is also a carbon atom supplier. The Ar and $\mathrm{C}_{2} \mathrm{H}_{2}$ were both made available in larger quantities for the sample denoted as $\mathrm{B}$ compared 
to sample A. It appears that in sample A the final graphene sheets arrangement in the spheres is less ordered (see Figures 1 and 2) and less Fe content is also found compared to sample B. Therefore, for sample A, the gas flux is sufficient to form the spheres but these are less graphitic and most amorphous. In practice, the $250 / 50 \mathrm{sccm} \mathrm{Ar} / \mathrm{C}_{2} \mathrm{H}_{2}$ gas flux adopted for sample $\mathrm{B}$ are better for synthesizing and separating carbon spheres of better graphitic character while keeping into account that the more Fe catalyst is present in the structure.

\subsection{Solar Cells}

In the last two decades, carbon nanomaterials (e.g., carbon nanotubes, fullerenes) with photogeneration properties have been exploited for solar energy conversion applications. ${ }^{[25]}$ One possible configuration employs the heterojunctions formed between materials with suitable band gaps in order to cover a wider range of the solar spectrum response. In particular, nanomaterial/semiconductor solar cells made of crystalline semiconductors, such as $\mathrm{Si}$, and carbon nanomaterials, rely on the cooperative action of $\mathrm{Si}$, that photogenerates electron-hole pairs and the nanomaterial (constituted of carbon) film that acts both as a photogenerator and as charge-transporting layer. ${ }^{[26]}$

Consequently, based on our experience, ${ }^{[26,27]}$ we developed a device to study the photon-energy conversion ability of the CS films deposited on a Si substrate, as illustrated in Figure S4 in the Supporting Information. The devices obtained from the deposition of the magnetic portion of samples A and B show a photocurrent response, while from the nonmagnetic part no significant results were found.

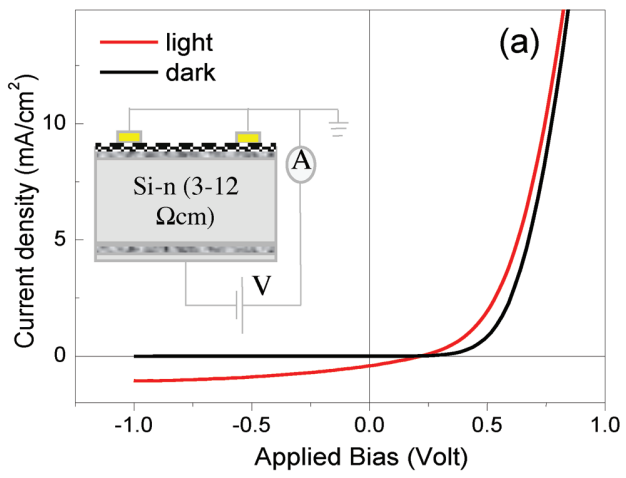

In particular, Figure 6 shows the electrical response of the CS/ Si best device, obtained from the sample $B_{m}$, in the dark state and under illumination (light state), enlarged in Figure $6 \mathrm{~b}$. The equivalent circuit diagram is the inset of Figure 6a. From the analysis of the light state curve, the short-circuit current density $J_{\mathrm{sc}}=0.42 \pm 0.05 \mathrm{~mA} \mathrm{~cm}^{-2}$ and the open circuit voltage $V_{\mathrm{oc}}$ is about $0.21 \pm 0.01 \mathrm{~V}$. The power conversion efficiency (PCE) achieved is about $1.2 \% \pm 0.2 \%$, with a fill factor $(\mathrm{FF})=0.3$ (Figure $6 \mathrm{c}$ ).

The obtained results are markedly below what our group found for single as well as multiwalled carbon nanotubes employed in similar devices. ${ }^{[26,27]}$ Nevertheless, the investigated CS-Si heterostructures show a light energy conversion ability in all the spectral range explored with a higher response in the visible range (Figure 6c).

The devices prepared with the magnetic samples gave a response and $\mathrm{B}_{\mathrm{m}}$ is the best. This last finding is very interesting and although we are still investigating on this point, we can gather some preliminary statements. There are some differences in the two CS samples that we can briefly summarize as follows: 1) the average diameter is similar but the graphene layers inside the spheres are more disordered in sample A compared to sample $\mathrm{B} ; 2$ ) the spheres in sample $\mathrm{B}$ tend to be more aggregated than sample $A ; 3)$ the sample $B_{m}$ has higher iron content than sample $A_{m}$, and the iron is present in metallic and oxidized state. These structural differences strongly influence the observed light response. The CS films constitute a disordered 2D network of variable conductivity. This variation originates from the presence of high-conductivity clusters separated by small low-conductivity ones. The CSs in the clusters are randomly arranged with several contacts and with local defects that act as electronic energy barriers, limiting the conduction.

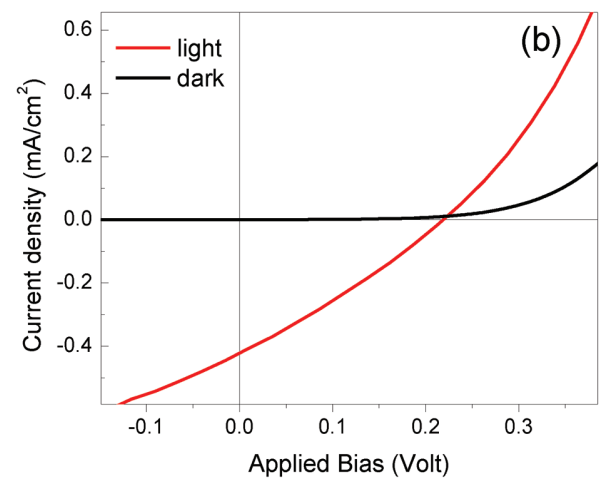

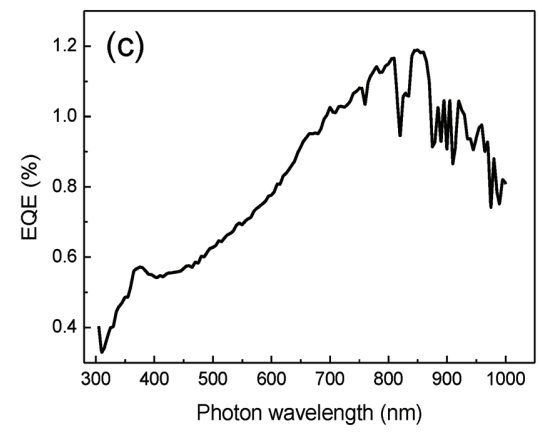

Figure 6. Device characterization. a) Electrical response of the best CS/Si device under dark (black line) and under illumination (red line). Inset: equivalent circuit diagram. b) Enlargement of the response curves reported in (a). c) External quantum efficiency (EQE). 
Therefore, since the photoelectrons produced upon light irradiation are weakly localized, percolate between the conducting regions by thermally induced hopping, and tunnel through less-conducting regions. This description reasonably supports the best response found for sample B (that showed the more ordered inner and outer structure favoring the conduction), with respect to A, and for magnetic samples, with respect to nonmagnetic ones. In addition, the observed CSs propensity to arrange in a necklace configuration in touch with each other facilitates the electron flow and collection in the device. The presence of iron inside the structure improves the photoresponse since it makes available additional charges and helps the conduction process. This behavior has been already found and described in detail for multiwalled carbon nanotubes. ${ }^{[28-31]}$

\subsection{Electrochemical Sensors}

In the electrochemical field, the exploitation of nanomaterials has boosted the tailored and high performant sensor and biosensor production thanks to physical, chemical, and catalytic properties of nanoparticles. ${ }^{[32-35]}$ The advantage of decreasing scale in electrochemistry can be ascribed to enhanced mass transport as well as reduced capacitive charging, ${ }^{[36]}$ conferring to the nanomodified sensors and biosensors improved electroanalytical performances in terms of electron transfer, sensitivity, working, and storage stability, to name a few. Among the carbonaceous materials, carbon nanotubes and graphene are the most employed in the electrochemical sensors and biosensors development, ${ }^{[37,38]}$ while few papers report on CSs in electrochemical devices. ${ }^{[39-45]}$ In addition, none of these papers investigated the CSs electrochemical behavior in terms of magnetic and nonmagnetic properties. For this reason, we directly evaluated the electrochemical performance of the CSs by drop casting a dispersion of CSs on electrochemical chips (E-chips) and analyzing the response toward several clinically relevant analytes. The E-chips were modified using magnetic and nonmagnetic CSs and tested toward ascorbic acid, cysteine, beta-Nicotinamide adenine dinucleotide (NADH), and dopamine analytes.

In detail, we investigated the electrochemical behavior of E-chips modified with the dispersion of $A_{m}, A_{n m}, B_{m}$, and $B_{n m}$, and we observed repeatable results only in the case of $B_{m}$ and $B_{n m}$. Consequently, Figure 7 only reports the cyclic voltammetries of the analytes tested using E-chip modified with $\mathrm{B}_{\mathrm{nm}}$ (red line) and with $\mathrm{B}_{\mathrm{m}}$ (green line). Furthermore, to better understand the effect on the E-chip modification, cyclic voltammetries using unmodified E-chip are reported (dashed black line). In the case of reversible behavior, as in the case of dopamine, we observed a significant decrease of peak to peak separation using E-chip modified with the dispersion $\mathrm{B}_{\mathrm{m}}$, demonstrating a better electron transfer. In addition, an increase of intensity of anodic and cathodic peaks was observed, probably ascribed to the higher number of electroactive sites. In the case of irreversible behavior (i.e., ascorbic acid, cysteine, and NADH) we have observed a relevant decrease of potential for oxidative reaction, demonstrating the electrocatalytic properties of the $\mathrm{B}_{\mathrm{m}}$ material. Moreover, the cyclic voltammetries achieved in only phosphate buffer (working solution without the analyte) demonstrate low capacitive current, confirming the good conductivity of $\mathrm{B}_{\mathrm{m}}$ material. For understanding the reason of this behavior, we correlated the structure with the electrochemical response. The low repeatability of the A samples could be ascribed to the more disordered arrangement of the graphene layers inside the spheres (see Figure 2) in respect to the B samples which also showed a better repeatability (relative standard deviation (RSD)\% lower than 10\%).

Regarding the E-chip modified with $\mathrm{B}_{\mathrm{m}}$ and $\mathrm{B}_{\mathrm{nm}}$, we suppose that the higher electrochemical performances of that modified with $B_{m}$ are ascribed to the iron content present in the magnetic CSs, taking into account that some authors reported the importance of metal impurities in the electrocatalysis of carbon nanotubes and graphene materials. For instance, Compton group $^{[46]}$ highlighted that metal impurities may cause the observed "electrocatalysis" using carbon-nanotube-modified electrodes and Pumera group claimed that "metal-free" electrocatalysis of the oxygen reduction reaction is due to the metallic impurities present in graphene materials. ${ }^{[47]}$ We can conclude that the presence of iron in CSsc can cover a double aspect is able: 1) to confer the magnetic properties to the material as well as 2) to improve the electrochemical performances of the magnetic modified CSs E-chips.

For instance, Banks et al., ${ }^{[46]}$ highlighted that the electrocatalysis for carbon nanotube modified electrode is due to the metal impurities in the carbon nanotubes that most probably consist $\mathrm{Fe}_{2} \mathrm{O}_{3}$. Similar results have been obtained for graphene by Pumera and co-workers ${ }^{[7]}$ that observed that an oxidative treatment of graphite samples is not sufficient to get rid of the impurities associated to the chemically reduced graphene that alter the electrochemical properties significantly. To this regard, the iron present in the magnetic CSs improves the electrochemical performances of the modified E-Chip and, at the same time, confers to CSs magnetic properties which can be exploited to easily manage this carbon-based material.

\section{Conclusion}

This paper describes a tailored vapor assisted chemical deposition method to obtain carbon spheres of nanometric dimensions. The cost of the synthesis process is reasonable and the yield is very high with a reduced presence of side products. Changing some of the growth parameter during the synthesis allows obtaining spheres of different morphology and structure. Most importantly, the iron catalyst employed in the reaction confers a marked magnetic response at room temperature to portions of the synthesis products. Therefore, the samples shared into two halves magnetic and nonmagnetic are analyzed separately. The inner and outer structural features of the spheres and the active contribution of iron mark the difference in the photoresponse as well as in the electrochemical performance of the two samples. The results are interesting and clearly show that the presence of residual iron inside the spheres is not detrimental for some targeted applications. These promising outcomes deserve further experimental investigation in order to optimize the CSs structural properties together with a detailed theoretical description that is still lacking. The low intrinsic mass of the spheres recommends their possible use as components in composite materials. The abundance of defects makes them highly reactive, suggesting applications as catalyst 

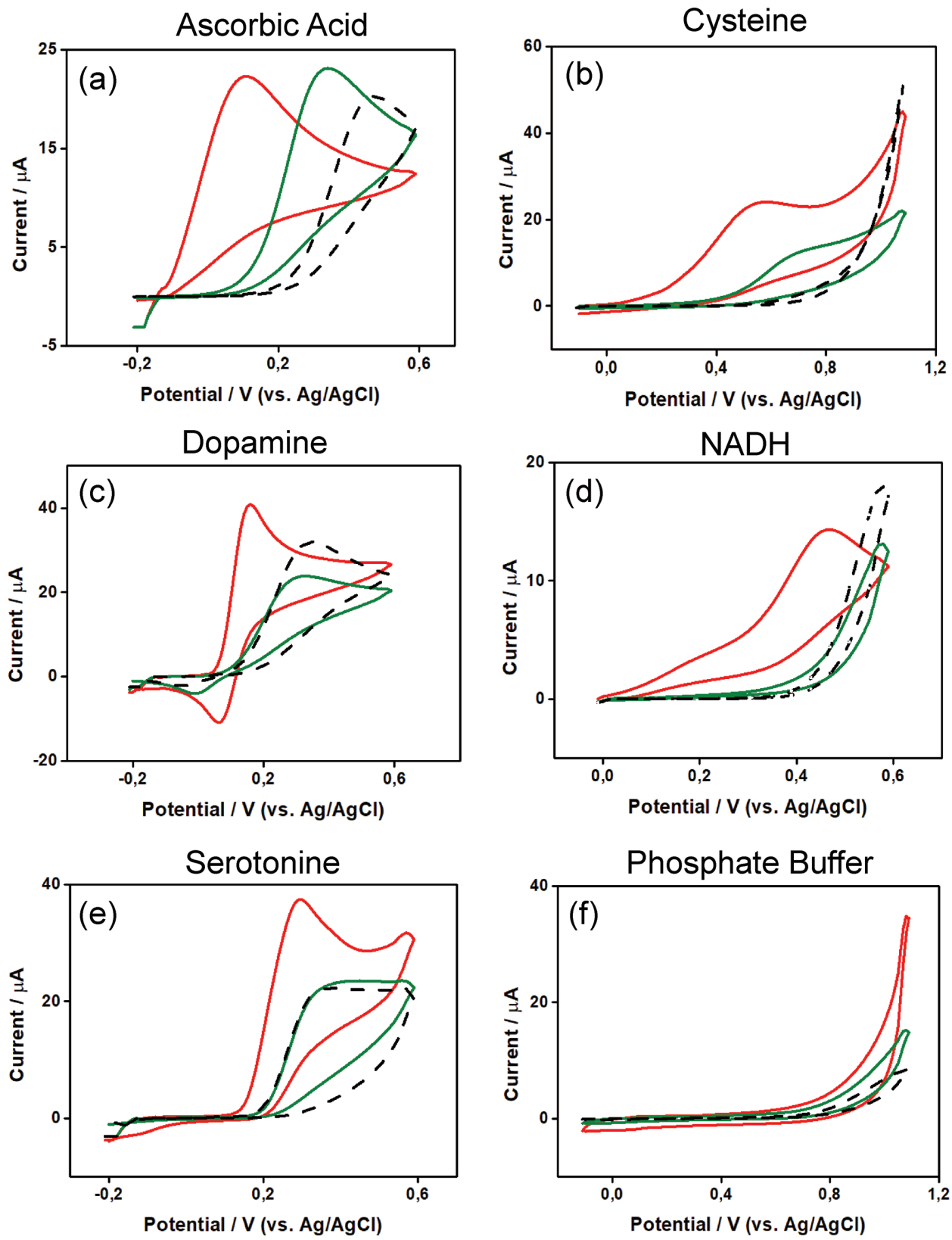

Figure 7. Cyclic voltammograms recorded in presence of $2 \times 10^{-3} \mathrm{M}$ of a) ascorbic acid, b) cysteine, c) dopamine, d) NADH, e) serotonin, and f) phosphate buffer. All the experiments were carried out in $0.05 \mathrm{M}$ phosphate buffer containing $0.1 \mathrm{~m}$ potassium chloride $(\mathrm{pH} 7.4)$ at a scan rate of $50 \mathrm{mV} \mathrm{s} \mathrm{s}^{-1}$ using bare E-chip (black dashed line) and E-chip modified with no magnetic CSs ( $B_{m}$, green line) or with magnetic CSs ( $B_{n m}$, red line).

support and the possibility of further surface chemical modifications in a wide range of chemical or biological systems.

\section{Experimental Section}

Preparation of Carbon Spheres: The carbon spheres were synthesized following a chemical vapor deposition process performed in a horizontal furnace. The furnace was first heated up to the growth temperature under argon atmosphere at ambient inert pressure (760 Torr). Ferrocene $\left(\mathrm{Fe}\left(\mathrm{C}_{5} \mathrm{H}_{5}\right)_{2}, 2.3 \mathrm{wt} \%\right)$, used as a catalyst, dissolved in dichlorobenzene at a concentration of $0.05 \mathrm{~g} \mathrm{~mL}^{-1}$. A glass syringe containing the solution injects drops of liquid at a constant rate $\left(5 \mathrm{~mL} \mathrm{~h}^{-1}\right)$ with the help of argon and acetylene gases that act as carrier and additional carbon precursor, respectively. The vaporized solution and the gas mixture enter into the chamber via a stainless pipe directly into a quartz tube placed in the hot region of the furnace kept at $800{ }^{\circ} \mathrm{C}$. The synthesis products collected from the tube appear in the form of black powder and small agglomerates. Two samples that differ in gas flux used during the reaction process were analyzed in this paper. The first was grown with argon/acetylene flux (50 and $25 \mathrm{sccm}$, respectively), named sample $A$ and the second with argon/acetylene flux $(250$ and $40 \mathrm{sccm}$, respectively), named sample B. No postgrowth purification processes were applied to the obtained powders.

Sample Characterization: The CS morphology and structure were examined using a field emission gun scanning electron microscopy (FEG-SEM, Leo Supra 35), equipped with energy dispersive X-ray, and 
a transmission electron microscopy (TEM, cold FEG Hitachi HF2000 operated at $200 \mathrm{kV}$ voltage). For the TEM analysis, the synthesis products were dispersed in ethanol ( $95 \%$ solution) and sonicated for half an hour. The samples were then dropped onto a copper grid (mesh 300) covered with a thin holey carbon film and dried before investigation.

XPS data were acquired using an ultrahigh vacuum PHI 1257 system equipped with a hemispherical analyzer, operating in the constant pass energy mode (with the total energy resolution of $0.8 \mathrm{eV}$ ) using a non-monochromatized $\mathrm{Mg} K_{\alpha}$ radiation source. The distance between the sample and the anode was about $40 \mathrm{~mm}$, the illumination area was about $1 \times 1 \mathrm{~cm}^{2}$ and the analyzed area was $400 \times 400 \mu \mathrm{m}^{2}$ with a take-off angle, between the sample surface and the photoelectron energy analyzer, of $45^{\circ}$. The energy scale was calibrated with reference to the binding energy of the $\mathrm{Cls}$ at $284.8 \mathrm{eV}$ with respect to the Fermi level.

The samples crystalline structure was investigated by XRD using a Siemens 5000 diffractometer with $\mathrm{Cu} \mathrm{K} \alpha$ radiation in Bragg-Brentano geometry. Joint Committee on Powder Diffraction Standards (JCPDS) cards 65-4899, 35-0772, and 41-1487 were used to index $\alpha-\mathrm{Fe}, \mathrm{Fe}_{3} \mathrm{C}$, and graphite- $2 \mathrm{H}$, respectively.

EELS, taken ex situ at room temperature on the samples, were recorded in the same $\mathrm{PHI} 1257$ system equipped with an electron gun $\left(E_{\mathrm{p}}=300 \mathrm{eV}, \Delta E=0.7 \mathrm{eV}\right)$. A room temperature micro-Raman spectra of the as-synthesized carbon spheres were recorded with an LABRAM spectrometer (Horiba-Jobin Yvon, $\lambda=633 \mathrm{~nm}, 1 \mu \mathrm{m}$ spatial resolution, and $\approx 2 \mathrm{~cm}^{-1}$ spectral resolution) equipped with a confocal optical microscope $(100 \times$ metallurgical plan achromat objective with 0.9 numerical aperture and $0.15 \mathrm{~mm}$ work distance).

Magnetic characterization was performed with an alternating gradient magnetometer (MicroMag 2900AGM, Princeton Measurements Corporation). Hysteresis loops were collected at room temperature with magnetic fields up to $1 \mathrm{~T}$ for as synthesized CSs and for selected partitions of the spheres obtained by magnetic separation using a permanent magnet, as explained in Section 2.1.

Photocurrent measurements were carried out at room temperature on CS films deposited on patterned silicon substrates (provided by Fondazione Bruno Kessler, Trento-Italy). CSs were dispersed in ethanol ( $30 \mathrm{mg} \mathrm{mL}^{-1}$ ) and ultrasonicated for one hour to improve the dispersion. Films of CS were fabricated following a vacuum filtration process of volume aliquots of the dispersion on cellulose filters (see Figure S3a, Supporting Information). The filters were cut and deposited by drytransfer printing technique described in detail in ref.[20] on the top of the device. The device consists of an $\mathrm{n}$-doped crystalline silicon covered by thermal $\mathrm{SiO}_{2}(300 \mathrm{~nm})$. The $\mathrm{SiO}_{2}$ has a patterned bare $\mathrm{Si}$ window $\left(2 \times 2 \mathrm{~mm}^{2}\right.$ ) delimited by $\mathrm{SiO}_{2}$ (the device active area is $0.04 \mathrm{~cm}^{2}$ ) with $\mathrm{Au} / \mathrm{Cr}$ back and front contact electrodes evaporated on $\mathrm{SiO}_{2}$ (see Figure S3b,c, Supporting Information). The light energy conversion ability of the films was tested using a solar simulator under air mass (AM) 1.5 spectral illumination (from LOT-Oriel, incident power $100 \mathrm{~mW} \mathrm{~cm}{ }^{-2}$ ). The output power density was calibrated using a power meter.

Electrochemical Sensor-Electrochemical Chip Fabrication: Electrochemical chips (E-chip) were produced with a 245 DEK (Weymouth, UK) screen-printing machine. Graphite-based ink (Electrodag 423 SS) from Acheson (Milan, Italy) was used to print both the working and auxiliary electrode. Silver/silver chloride ink (Electrodag 477 SS) was used to print the pseudo-reference electrode. The substrate has been a flexible polyester film (Autostat HT5) purchased from Autotype Italia (Milan, Italy). The diameter of the working electrode was $0.3 \mathrm{~cm}$ resulting in a geometric area of $0.07 \mathrm{~cm}^{2}$.

Electrochemical Sensor-Preparation of CSs Dispersions: The produced CS powders were dispersed in a hydro/organic media. $10 \mathrm{mg}$ of the selected powders were first dissolved in $5 \mathrm{~mL}$ of $\mathrm{N}, \mathrm{N}$ dimethylformammide and subsequently $5 \mathrm{~mL}$ of distilled water were added. The mixture was sonicated for $1 \mathrm{~h}$ at $59 \mathrm{kHz}$. The dispersion was stored in the dark at room temperature.

Electrochemical Sensor-Procedure for E-chip Modification: E-chip was modified by drop casting $2 \mu \mathrm{L}$ of the dispersion onto the working electrode surface. The modified E-chips were interrogated after the solvent of the dispersion completely evaporated.

\section{Supporting Information}

Supporting Information is available from the Wiley Online Library or from the author.

\section{Acknowledgements}

M.S. and M.D.C. acknowledge European Community for the Research, Innovation and Science Policy Experts (RISE) Project CoExAN GA644076. F.A. acknowledges European Community for the ERANETMED NanoSWS Project.

\section{Conflict of Interest}

The authors declare no conflict of interest.

\section{Keywords}

chemical vapor deposition, electrochemical response, magnetic properties, photon-energy conversion, structural properties

Received: January 15, 2018

Revised: March 29, 2018

Published online:

[1] P. W. Fowler, D. E. Manolopoulos, An Atlas of Fullerenes, Oxford University Press, New York 1995.

[2] A. Krueger, Carbon Materials and Nanotechnology, Wiley-VCH Verlag $\mathrm{GmbH} \&$ Co. KGaA, Weinheim 2010.

[3] Z. L. Wang, Z. C. Wang, J. Phys. Chem. 1996, 100, 17725.

[4] K. Yamada, S. Tobisawa, Carbon 1989, 27, 845.

[5] M. L. Studebaker, Rubber Chem. Technol. 1957, 30, 1400.

[6] A. A. Deshmukh, S. D. Mhlanga, N. J. Coville, Mater. Sci. Eng. $R$ 2010, 70, 1 .

[7] A. Nieto-Márquez, R. Romero, A. Romero, J. L. Valverde, J. Mater. Chem. 2011, 21, 1664.

[8] M. Inagaki, Carbon 1997, 31, 711.

[9] P. Serp, R. Feurer, P. Kalck, Y. Kihn, J. L. Faria, J. L. Figueiredo, Carbon 2001, 39, 621.

[10] J. Qiu, Y. Li, Y. Wang, C. Liang, T. Wang, D. Wang, Carbon 2003, 41, 767.

[11] Y. Ma, Z. Hu, K. Huo, Y. Lu, Y. Hu, Y. Liu, J. Hu, Y. Chen, Carbon 2005, 43, 1667

[12] H.-S. Qian, F.-M. Han, B. Zhang, Y.-C. Guo, J. Yue, B.-X. Peng, Carbon 2004, 42, 761.

[13] V. V. Kovaleski, A. N. SafronovCarbon 199836, 963.

[14] P. E. Nolan, D. C. LynchCarbon 199634, 817.

[15] Z. C. Kang, Z. L. Wang, J. Mol. Catal. A: Chem. 1997, 118, 215.

[16] M. Inagaki, K. Kuroda, M. SakaiCarbon 1983, 21, 231.

[17] B. Basavalingu, J. M. Calderon Moreno, K. Byrappa, Y. G. Gogotsi, M. Yoshimura, Carbon 2001, 39, 1763.

[18] X.-Y. Liu, B. Huang, N. J. Coville, Carbon 2002, 40, 791.

[19] J.-Y. Miao, D. W. Hwang, K. V. Narasimhulu, P.-I. Lin, Y.-T. Chen, S.-H. Lin, L.-P. Hwang, Carbon 2004, 42, 813.

[20] J. L. Dormann, D. Fiorani, E. Tronc, Advances in Chemical Physics, Vol. 98 (Eds: I. Prigogine, S. A. Rice), Wiley, New York 1997, p. 283.

[21] P. Zhang, X. Wang, W. Wang, X. Lei, H. Yang, RSC Adv. 2015, 5, 21670. 
[22] S. V. Komogortsev, R. S. Iskhakov, A. D. Balaev, A. G. Kudashow, A. V. Okotrub, S. I. Smirnov, Phys. Solid State 2007, 49, 734.

[23] E. C. Vermisoglou, G. Pilatos, G. E. Romanos, E. Devlin, N. K. Kanellopoulos, G. N. Karanikolos, Nanotechnology 2011, 22, 355602.

[24] F. S. Boi, S. Maugeri, J. Guo, M. Lan, S. Wang, J. Wen, G. Mountjoy, M. Baxendale, G. Nevill, R. M. Wilson, Y. He, S. Zhang, G. Xiang, Appl. Phys. Lett. 2014, 105, 243108.

[25] D. D. Tune, B. S. Flavel, R. Krupke, J. G. Shapter, Adv. Energy Mater. 2012, 2, 1043.

[26] S. Del Gobbo, P. Castrucci, S. Fedele, L. Riele, A. Convertino, M. Morbidoni, F. De Nicola, M. Scarselli, L. Camilli, M. De Crescenzi, J. Mater. Chem. C 2013, 1, 6752.

[27] F. De Nicola, M. Salvato, C. Cirillo, M. Crivellari, M. Boscardin, M. Scarselli, F. Nanni, I. Cacciotti, M. De Crescenzi, P. Castrucci, Carbon 2016, 101, 226.

[28] M. Scarselli, L. Camilli, P. Castrucci, F. Nanni, S. Del Gobbo, E. Gautron, S. Lefrant, M. De Crescenzi, Carbon 2012, 50, 875

[29] M. Scarselli, C. Scilletta, F. Tombolini, P. Castrucci, M. Diociaiuti, S. Casciardi, E. Gatto, M. Venanzi, M. De Crescenzi, J. Phys. Chem. C 2009, 113, 5860.

[30] M. Scarselli, C. Scilletta, F. Tombolini, P. Castrucci, M. Diociaiuti, S. Casciardi, F. Tombolini, E. Gatto, M. Venanzi, M. De Crescenzi, Nanotechnology 2011, 22, 035701.

[31] M. Scarselli, L. Camilli, L. Matthes, O. Pulci, P. Castrucci, E. Gatto, M. Venanzi, M. De Crescenzi, Appl. Phys. Lett. 2012, 101, 241113.
[32] F. Arduini, S. Cinti, V. Scognamiglio, D. Moscone, G. Palleschi, Anal. Chim. Acta 2017, 959, 15

[33] F. Arduini, S. Cinti, V. Scognamiglio, D. Moscone, Microchim. Acta 2016, 183, 2063

[34] C. Zhu, G. Yang, H. Li, D. Du, Y. Lin, Anal. Chem. 2014, 87, 230.

[35] A. Chen, S. Chatterjee, Chem. Soc. Rev. 2013, 42, 5425.

[36] P. H. Robbs, N. V. Rees, Phys. Chem. Chem. Phys. 2016, 18, 24812.

[37] S. Cinti, F. Arduini, Biosens. Bioelectron. 2017, 89, 107.

[38] Q. Cao, J. A. Rogers, Adv. Mater. 2009, 21, 29.

[39] Q. Xu, F. Yan, J. Lei, C. Leng, H. Ju, Chem. Eur. J. 2012, 18, 4994.

[40] T. Gan, Z. Lv, J. Sun, Z. Shi, Y. Liu, J. Hazard. Mater. 2016, 302, 188.

[41] B. K. Mutuma, R. Rodrigues, K. Ranganathan, B. Matsoso, D. Wamwangi, I. A. Hümmelgen, N. J. Coville, J. Mater. Chem. A 2017, 5, 2539.

[42] L. Kong, X. Lu, X. Bian, W. Zhang, C. Wang, Langmuir 201026, 5985.

[43] X. Bian, K. Guo, L. Liao, J. Xiao, J. Kong, C. Ji, B. Liu, Talanta 2012, 99, 256

[44] S. P. Dubey, A. D. Dwivedi, I.-C. Kim, M. Sillanpaa, Y.-N. Kwon, C. Lee, Chem. Eng. J. 2014, 244, 160.

[45] J. Zhang, J. Ma, S. Zhang, W. Wang, Z. Chen, Sens. Actuators, B 2015, 211, 385

[46] C. E. Banks, A. Crossley, C. Salter, S. J. Wilkins, R. G. Compton, Angew. Chem., Int. Ed. 2006, 45, 2533.

[47] L. Wang, A. Ambrosi, M. Pumera, Angew. Chem., Int. Ed. 2013, 52, 13818. 